\title{
Time series study of EUV spicules observed by SUMER/SoHO
}

\author{
L. D. Xia ${ }^{1,2}$, M. D. Popescu ${ }^{2,3}$, J. G. Doyle ${ }^{2}$, and J. Giannikakis ${ }^{2,4}$ \\ 1 CAS Key Laboratory of Basic Plasma Physics, School of Earth and Space Sciences, \\ Univ. of Science and Technology of China, Hefei, Anhui 230026, PR China \\ e-mail: xld@ustc.edu.cn \\ 2 Armagh Observatory, College Hill, Armagh BT61 9DG, N. Ireland \\ e-mail: [mdp; jgd]@arm.ac.uk \\ 3 Astronomical Institute of the Romanian Academy, 75212 Bucharest 28, Romania \\ ${ }^{4}$ Sect. of Astrophysics, Astronomy and Mechanics, Dept. of Physics, Univ. of Athens, Athens 15783, Greece
}

Received 21 December 2004 / Accepted 18 April 2005

\begin{abstract}
Here we study the dynamic properties of EUV spicules seen at the solar limb. The selected data were obtained as time series in polar coronal holes by SUMER/SoHO. The short exposure time and the almost fixed position of the spectrometer's slit allow the analysis of spicule properties such as occurrence, lifetime and Doppler velocity. Our data reveal that spicules occur repeatedly at the same location with a birth rate of around $0.16 / \mathrm{min}$ as estimated at $10^{\prime \prime}$ above the limb and a lifetime ranging from 15 down to $\approx 3 \mathrm{~min}$. We are able to see some spicules showing a process of "falling after rising" indicated by the sudden change of the Doppler velocity sign. A periodicity of $\approx 5 \mathrm{~min}$ is sometimes discernible in their occurrence. Most spicules have a height between $10^{\prime \prime}$ and $20^{\prime \prime}$ above the limb. Some can stretch up to $40^{\prime \prime}$; these "long macro-spicules" seem to be comprised of a group of high spicules. Some of them have an obvious periodicity in the radiance of $\approx 5 \mathrm{~min}$.
\end{abstract}

Key words. Sun: UV radiation - Sun: transition region

\section{Introduction}

Solar spicules were seen on the limb more than a century ago (Secchi 1877; Roberts 1945). They are rapidly evolving jetlike structures extending from the chromosphere upward into the corona, and are probably the most common feature of the upper solar atmosphere. Spicules were traditionally defined in the cool chromospheric lines such as $\mathrm{H} \alpha$. Reviews summarizing $\mathrm{H} \alpha$ spicule properties have been given by Beckers (1968, 1972) and recently by Sterling (2000). Based on $\mathrm{H} \alpha$ observations (Beckers 1968, 1972, and references therein), typically, spicules have an average length ranging from $6500 \mathrm{~km}$ to $9500 \mathrm{~km}$, widths from several hundred to $2000 \mathrm{~km}$ and lifetimes from several minutes to $15 \mathrm{~min}$. They have an apparent upward velocity of about $25 \mathrm{~km} \mathrm{~s}^{-1}$. After having reached the maximum height, spicules are found either falling back into the chromosphere or fading out in the transition region (TR). Spicules are reported to be slightly taller and oriented more nearly vertical in coronal holes $(\mathrm{CHs})$, and are somewhat more numerous at polar than at low latitudes. Spicules are estimated to cover about $1 \%$ of the Sun's surface. Their typical chromospheric density is $\approx 10^{11} \mathrm{~cm}^{-3}$. The upward mass flux in spicules is estimated to be two orders of magnitude larger than that needed to maintain the solar wind. Yet, spicules are still among the most unresolved solar phenomena, as their physical conditions are difficult to be clearly defined from observations due to their small size and duration.
Since the launch of satellites, we are also able to observe spicule-like structures above the limb in UV and EUV wavelengths. Bohlin et al. (1975) were the first to clearly notice He II jets at the solar limb with the help of the XUV spectrograph on the Skylab mission. In their data, such jets were found to construct a nearly continuous band over the polar caps, ranging from $5^{\prime \prime}$ to over $60^{\prime \prime}$ in length $\left(1^{\prime \prime} \approx 720 \mathrm{~km}\right.$ on the Sun), from $5^{\prime \prime}$ to $30^{\prime \prime}$ in width, and from 5 to over $40 \mathrm{~min}$ in lifetime. It should be noted that the term "macrospicules" was then used by Bohlin et al. to name the newly discovered EUV spicules in order to distinguish them from the $\mathrm{H} \alpha$ spicules. In this context, the name "macrospicule" is independent of the actual length of EUV spicules. In the following sections, we use the term "long macro-spicule" for these EUV spicules that were occasionally observed as high as $30000 \mathrm{~km}$ off-limb. Note that, in the case of long $\mathrm{H} \alpha$ spicules, the term "H $\alpha$ macrospicule" is used (see, e.g., Moore et al. 1977).

From the Naval Research Laboratory (NRL) High Resolution Telescope and Spectrograph (HRTS) and the Harvard experiment on Skylab, more information about EUV spicules emerged (see, e.g., Withbroe 1983; Dere et al. 1983, 1989; Karovska \& Habbal 1994). Nevertheless, EUV spicules are more difficult to observe than the $\mathrm{H} \alpha$ ones, not only because the spatial resolution needed to study spicules is more difficult to achieve with EUV instruments in space, but also because they are much harder to detect against the bright TR background. 
Table 1. SUMER observations of temporal series in polar coronal holes (PCHs).

\begin{tabular}{|c|c|c|c|c|c|c|}
\hline Item & Date & $\begin{array}{l}\text { Time } \\
\text { UT }\end{array}$ & $\begin{array}{c}\text { Solar coordinate } \\
x\left({ }^{\prime \prime}\right), y\left({ }^{\prime \prime}\right)\end{array}$ & $\begin{array}{l}\text { Wavelength used } \\
\text { (Å) }\end{array}$ & $\begin{array}{l}\text { Detector } \\
\text { and slit }\end{array}$ & $\begin{array}{c}\text { Exposure } \\
\text { time (s) }\end{array}$ \\
\hline PCH 1 & $20 / 10 / 1996$ & $19: 57-23: 57$ & $0,909.75 \mathrm{~N}$ & N IV $765 \AA$, Ne VIII $770 \AA$ & B $2\left(1^{\prime \prime} \times 300^{\prime \prime}\right)$ & 30 \\
\hline $\mathrm{PCH} 2 \mathrm{a}$ & $25 / 02 / 1997$ & $00: 03-13: 58$ & $0,950.25 \mathrm{~S}$ & N IV $765 \AA$ & B $2\left(1^{\prime \prime} \times 300^{\prime \prime}\right)$ & 60 \\
\hline $\mathrm{PCH} 2 \mathrm{~b}$ & $21 / 02 / 1997$ & $01: 20-01: 35$ & $0,984.75 \mathrm{~S}$ & C III $977 \AA$ & B $6\left(0.3^{\prime \prime} \times 120^{\prime \prime}\right)$ & 15 \\
\hline
\end{tabular}

The best spectral and spatial resolution currently available in the EUV for solar studies comes from SUMER (Solar Ultraviolet Measurements of Emitted Radiation), a spectrometer on SoHO (the Solar and Heliospheric Observatory). Its spatial resolution of $1^{\prime \prime}$, together with a high spectral resolution and a time cadence of a few seconds, makes it a good instrument for resolving small-scale and rapidly evolving structures such as spicules.

According to SUMER observations (Budnik et al. 1998; Wilhelm et al. 2000; Wilhelm 2000), EUV spicules have a length comparable to $\mathrm{H} \alpha$ spicules. They have widths of about 1-2" when seen at chromospheric temperatures, and are wider in TR lines. Their enhanced radiance structures can be distinguishable up to a temperature of about $2 \times 10^{5} \mathrm{~K}$. The line-of-sight (LOS) velocity derived from the Doppler shift has average values of $\pm 30 \mathrm{~km} \mathrm{~s}^{-1}$.

Spicule studies are still in their infancy, and many questions about their properties are not yet answered, not to mention the efforts which are being made for finding the mechanism that is producing them. There is a strong debate as to whether they are due to propagating waves or to magnetic reconnection.

Another one of the most controversial issues is the connection between $\mathrm{H} \alpha$ and EUV spicules. Although both features look like thin elongated jets, a direct comparison is rather difficult (see, e.g., discussion in Sterling 2000). Because the density of EUV spicules measured at TR temperatures is much lower and their width is much broader than those of $\mathrm{H} \alpha$ spicules, it is suggested that, if these two structures belong to the same phenomenon, the EUV spicules are likely the hot sheath of the cool $\mathrm{H} \alpha$ spicules, which constructs a transition between the hotter corona and the cooler material from below (Withbroe 1983).

In the attempt to elucidate more of the spicule characteristics, in this contribution we study the dynamic properties of EUV spicules. The data selected for this study were obtained as time series in polar CHs by SUMER. The short exposure time (compared with the lifetime of spicules) and the almost fixed position (due to a reduced effect of the solar rotation at the poles) allow us to analyse the spicule occurrence, lifetime, LOS velocity and other characteristics derived from the good spectral information of our data. The temporal evolution of solar EUV spicules has never previously been seen for such long observing sequences or with such clarity as reported here. A colour version of the figures from this paper is given on-line.

\section{Observations and data analysis}

The data selected for this study were obtained as time series in two polar coronal holes (PCHs, see Table 1) by the SUMER
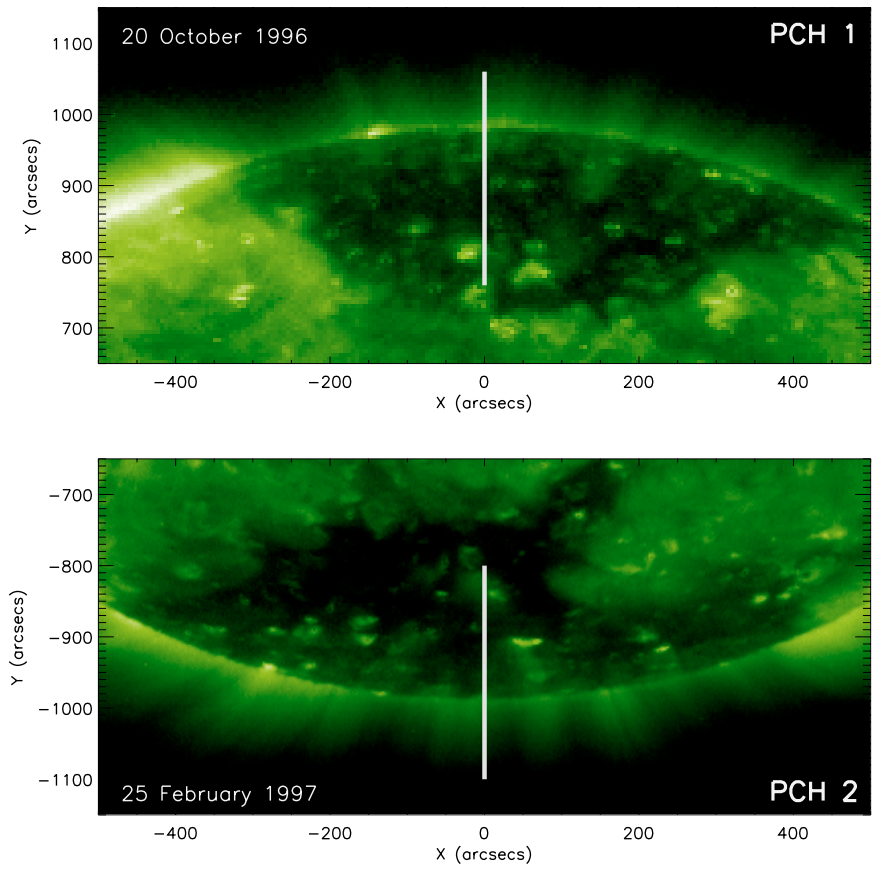

Fig. 1. The two figures illustrate the solar poles in the light emitted by the Fe XII $195 \AA$ line $\left(1.5 \times 10^{6} \mathrm{~K}\right)$, taken by EIT/SoHO. The vertical white lines show the fixed position of the SUMER slit during the observations for PCH 1 and PCH 2a. The third dataset, $\mathrm{PCH} 2 \mathrm{~b}$, is taken in the same southern hole, four days before the PCH 2a data.

spectrometer (Wilhelm et al. 1995, 1997; Lemaire et al. 1997). During the observation, the SUMER slit was fixed (without scanning) at position $x=0^{\prime \prime}, y=909.75^{\prime \prime}$ for PCH $1, x=0^{\prime \prime}$, $y=-950.25^{\prime \prime}$ for PCH 2a, and $x=0^{\prime \prime}, y=-984.75^{\prime \prime}$ for PCH 2b. The two CHs are shown in Fig. 1, where the EIT (Extreme Ultraviolet Imaging Telescope) $195 \AA$ images are plotted overlaid with the position of the SUMER slit. The two PCHs (dark regions) can be clearly distinguished in the images.

The slit width ( 1 " for PCH 1 and PCH 2a; $0.3^{\prime \prime}$ for PCH 2b) determine the spatial resolution along the $x$-direction, while the resolution element along the slit in the $y$-direction (north-south; positive towards north) is given by the pixel size of the detector, and is approximately $1^{\prime \prime}$. The exposure time for each dataset was: $30 \mathrm{~s}$ for PCH 1, $60 \mathrm{~s}$ for PCH 2a, and $15 \mathrm{~s}$ for PCH $2 \mathrm{~b}$.

In our analysis, we first applied the standard procedures for correcting and calibrating the SUMER raw data. They include decompression, reversal, as well as flat-field, dead-time, localgain and geometrical corrections. The method used to deduce the line parameters (radiance, central position of the spectral 

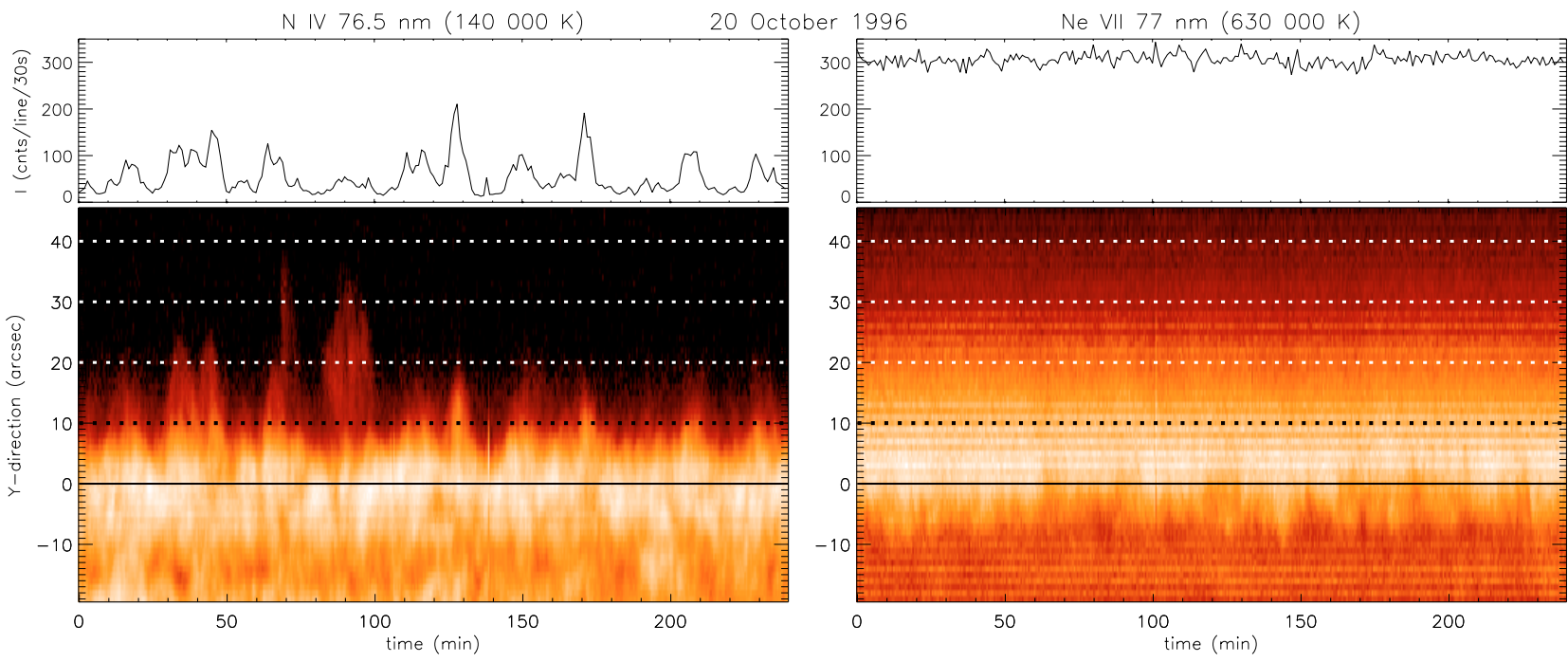

Fig. 2. The temporal evolution of the northern coronal hole (PCH 1) seen in the N IV $765 \AA$ A (left) and Ne VIII $770 \AA$ A (right) lines, with a cadence of $30 \mathrm{~s}$. Spicule structures are clearly seen in the TR line N IV $(140000 \mathrm{~K})$, but not in the higher temperature line Ne VIII $(630000 \mathrm{~K})$. At the top of the figures we show the radiance varying with time for both lines at the height of $10^{\prime \prime}$ above the limb (indicated, in the $2 \mathrm{D}$ plots, by the dotted black line). The continuous black line represents the N IV limb.

line and width) is described as follows. We calculated the central position for every pixel, by integrating the line radiance across a certain spectral window and determining subsequently the location of the $50 \%$ level with sub-pixel accuracy. This procedure is frequently used to obtain SUMER Dopplergrams (see details in Dammasch et al. 1999) and dramatically reduces the computing time for a large number of data. The results deduced by this method are statistically consistent with those obtained by using the standard Gaussian fitting programme (Xia 2003). In addition, a line-position correction was applied, to remove spurious spectral line shifts caused by thermal deformations of the instrument, and to eliminate residual errors (systematically varying along the slit) after the geometric correction, using the standard software (Dammasch et al. 1999; Xia 2003).

\section{Occurrence, length and lifetime}

The time evolution of a small region at the solar limb, inside the northern coronal hole (PCH 1), is displayed in Fig. 2 as radiance maps in the N IV $765 \AA$ (left) and Ne VIII $770 \AA$ (right) lines. The maps show a time sequence taken repeatedly every $30 \mathrm{~s}$ for $\approx 4 \mathrm{~h}$. In order to increase the visibility of structures, we have processed this image by using a logarithmic scale to improve its contrast.

The limb position is set as $Y=0$. We have determined it as the peak-radiance position along the solar $y$-direction in the N IV line. Previous studies (see Mariska 1992) indicate that the white-light limb would be about $2^{\prime \prime}$ lower than this. And it is indeed $2^{\prime \prime}$ lower if we look at the continuum in our data.

At the top of the figures, we plot the radiance varying with time at a height of $10^{\prime \prime}$ above the limb. One finds that spicule structures are clearly seen in the N IV TR line due to the high brightness contrast between spicules and the dark regions, while they are indistinguishable in the Ne VIII line. Further inspection of the latter line reveals that its time-dependent radiance variation above a height of $5^{\prime \prime}$ falls in a range of standard deviation, indicating that the temperature of spicules cannot reach $6 \times 10^{5} \mathrm{~K}$, the value of the Ne VIII formation temperature. This is consistent with previous observations (Withbroe 1983; Budnik et al. 1998; Wilhelm et al. 2000). In the following study, we only use the N IV line due to its strong radiance and almost pure profile without blends of other lines.

In Fig. 3, we show spicule structures observed in $\mathrm{PCH} 2 \mathrm{a}$ in the N IV line, with a cadence of one minute, for almost $14 \mathrm{~h}$. The figure shows solar spicules continuously appearing and then fading away at the same location, allowing us to see the spicule evolution with clarity and for a long single observing sequence.

Note that both Figs. 2 and 3 display time-series radiance maps, which are different than raster images. Spicule shapes in such a map exhibit their evolution with time if they can be detected at the location where the SUMER slit is pointed. Therefore, the shapes do not reflect a spatial expansion along the $x$-direction. Nevertheless, we are able to see how they expand along the $y$-direction (radial direction in these observations) and how long they persist in the field of view (lifetime). In this context, a narrow (wide) feature seen in such an image means it has a short (long) lifetime. In addition, different shapes may represent different evolution stages of spicules, e.g., in Fig. 3 the expansion front tilts towards the right side when they are rising and towards the left when falling.

From Figs. 2 and 3, one can see that most spicules have a height between $10^{\prime \prime}$ and $20^{\prime \prime}$ (similar in range to a typical $\mathrm{H} \alpha$ spicule length). Nevertheless, some of them can reach heights of $30^{\prime \prime}-40^{\prime \prime}$ and can be identified as long macrospicules in terms of length.

An exact counting of spicules is difficult due to their different sizes and overlaps, in particular, for those with small lengths. However, an estimate can be made for those visible at a height above $10^{\prime \prime}$. Figure 4 displays the radiance ratio $I / I_{\mathrm{b}}$, in which $I_{\mathrm{b}}$ is defined as the background radiance taken from 


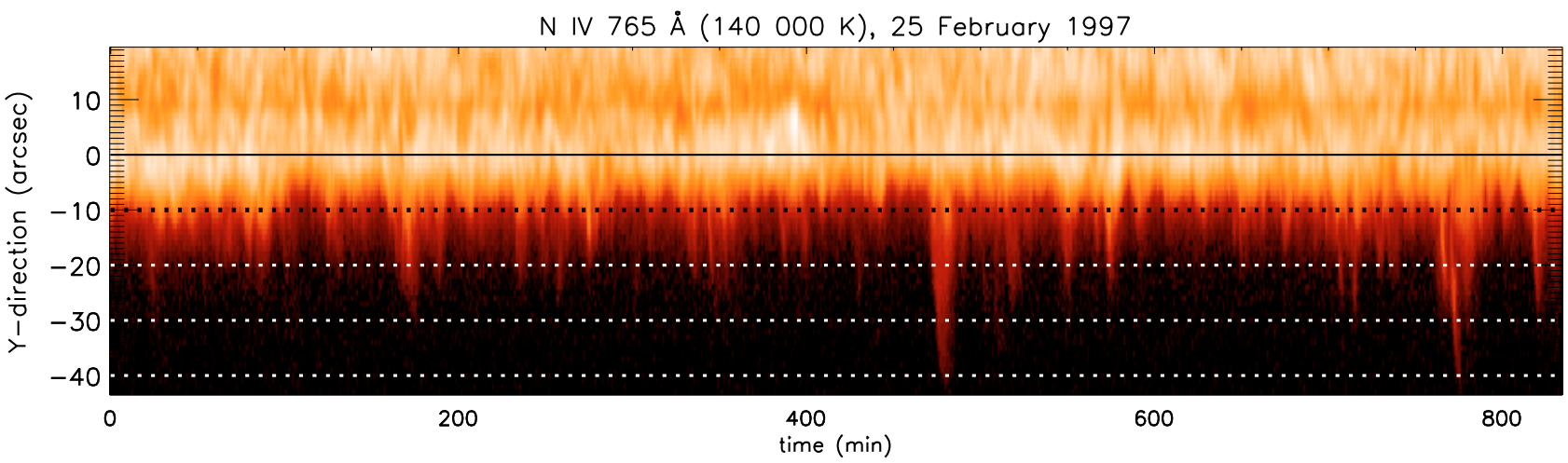

Fig. 3. The evolution of a small region at the solar limb, inside the southern coronal hole ( $\mathrm{PCH} 2 \mathrm{a})$. The image is a time sequence taken repeatedly every minute for $\approx 14 \mathrm{~h}$. Brighter colors represent higher radiance of the N IV $765 \AA$ Aline.

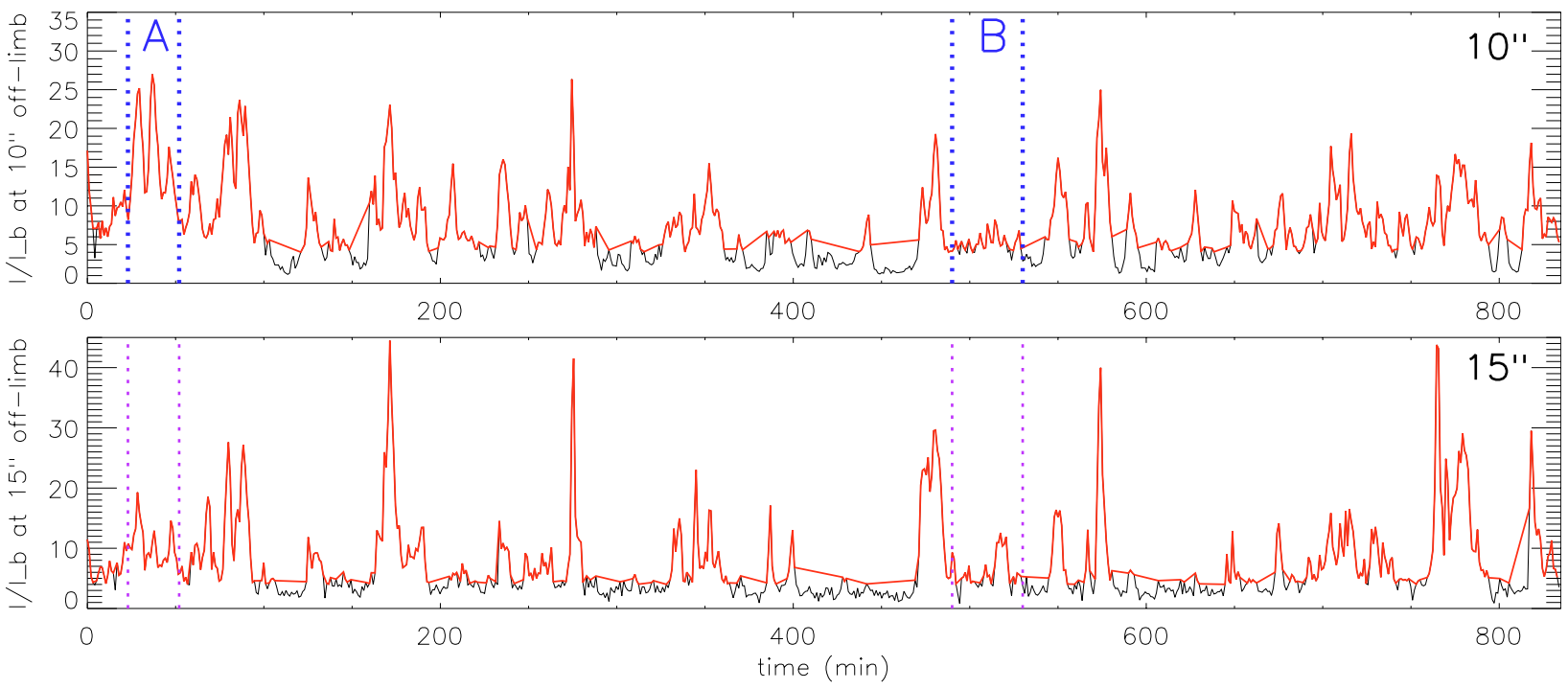

Fig. 4. Radiance ratio $I / I_{\mathrm{b}}\left(I_{\mathrm{b}}\right.$ is the background radiance taken from the darkest pixel at each height) versus time (in min) at $10^{\prime \prime}$ and $15^{\prime \prime}$ above the limb for PCH 2a (N IV $765 \AA$ line). The over-plotted curves represent $I_{\mathrm{b}} \geq 4$; we use them for counting the spicules. With " $\mathrm{A}$ " and "B" we denote two locations where spicules show an obvious periodicity.

the darkest pixel at each height, versus time, at $10^{\prime \prime}$ and $15^{\prime \prime}$ off-limb. The over-plotted curves represent the radiance ratio larger than 4, and are used to count the number of spicules. The occurrence rate of spicules during the entire observation, viewed by eye, is estimated to be about $0.16 / \mathrm{min}$, with little difference between $10^{\prime \prime}$ and $15^{\prime \prime}$ off-limb.

An average lifetime of about 5 min can be inferred from the occurrence rate discussed above. An inspection of individual structures indicates that their lifetimes range from $\approx 15$ down to $\approx 3 \mathrm{~min}$. This is in agreement with previous studies of H $\alpha$ spicules (Beckers 1972), as well as EUV spicules (see, e.g. Dere et al. 1989; Budnik et al. 1998). However, an exact measurement of the spicule lifetime is not easy, especially for those which are short-lived, because their roots are overlaid with each other, making them difficult to be determined individually.

Spicules are sometimes found to have a periodic occurrence, with periods ranging from 3-5 min to $10-15 \mathrm{~min}$. But as they are so dynamic, they look different along the distance from the limb. For example, one can see in the upper frame of Fig. 4 at location "A" a period of $\approx 10 \mathrm{~min}$, and at location "B" a period of $\approx 5 \mathrm{~min}$. Nevertheless, the situation changes higher up, at $15^{\prime \prime}$ : the structures from location "A" now show a finer detail, revealing that each of the three clear peaks from $10^{\prime \prime}$, "split" into two, showing a small peak in front of the big one. This could indicate that such a 10 min-living structure (as seen at $10^{\prime \prime}$ above the limb) is actually made up from two independent features, or that the spicular material is falling back to the surface after rising, having a slightly different form. Other locations in Fig. 4 also undergo obvious changes in the structure viewed at different heights off-limb. To further analyse the characteristics of spicules, we look at the Doppler shift of the plasma in these features.

\section{Velocity}

In Fig. 5, thin black lines in the bottom panels represent the Doppler velocity for the first 100 min taken from PCH 2a, with the radiance curve over-plotted as a thick grey line (in arbitrary units). Note that the radiance is inverted to enable an easier comparison with the contours showing different levels of radiance in logarithmic scale, from the temporal map in the top panel. We assume that the velocity is zero at the limb, therefore 

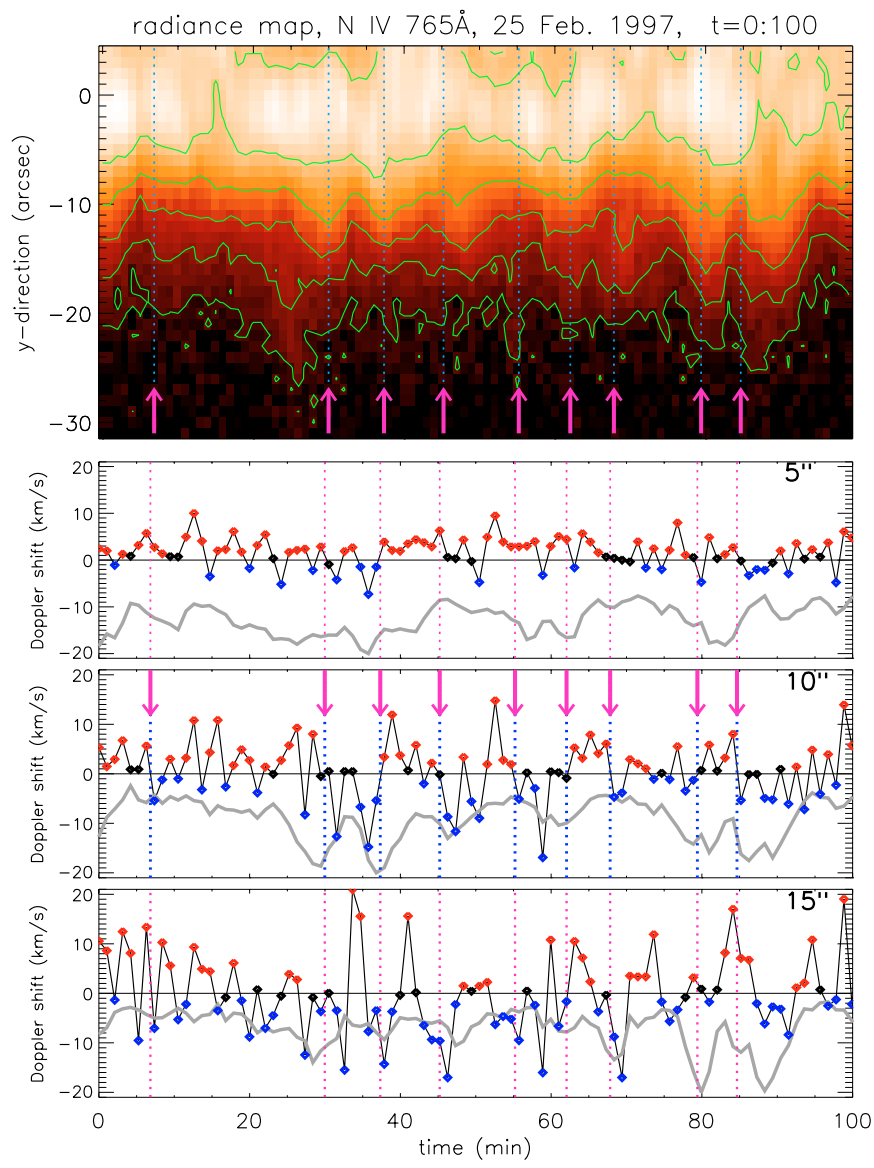

Fig. 5. Top panel: a segment plot (the first $100 \mathrm{~min}$ ) taken from $\mathrm{PCH} 2 \mathrm{a}$ showing the temporal map of the N IV $765 \AA$ radiance, with overlaid contours showing different levels of radiance in logarithmic scale. The other panels represent the line Doppler shift (thin black curve) and radiance (the thick grey curve) varying with time at three locations: at $5^{\prime \prime}, 10^{\prime \prime}$ and $15^{\prime \prime}$ above the limb. Note that the radiance is inverted to enable an easier comparison with the contours from the top panel. Vertical dotted lines show examples of positions where, in the third panel (at $\left.10^{\prime \prime}\right)$, the velocity changes its sign (negative values represent blue-shifts, or outflows; positive values - redshifts, or inflows). This transition of the spicule Doppler shift could be caused by material falling after rising.

we have subtracted $2 \mathrm{~km} \mathrm{~s}^{-1}$ from its values. This is, nevertheless, within the limit of the uncertainty for this type of data, which, in a previous study, we have estimated to be $\approx 3 \mathrm{~km} \mathrm{~s}^{-1}$ (Popescu et al. 2004).

Like the line radiance, the velocities deduced from line shift are highly structured, with a time scale down to a few minutes. In addition, they seem to vary with height if observed at the same time. Obviously, the higher we go off-limb, the more structure we see in spicules, and the wider become the "interspicular" gaps, with low plasma density. The precision of the Doppler shift measurement decreases with height, but not uniformly, as we can still measure it quite well inside the spicules.

To see where the changes in the velocity sign are taking place, we show some of these examples as vertical dotted lines in Fig. 5; they mark out the position at $10^{\prime \prime}$ above the limb (the third panel) where the Doppler velocity changes the sign. Following the radiance curves at $10^{\prime \prime}$ and $15^{\prime \prime}$ off-limb in the one-dimensional plots or in the 2D temporal radiance map (top panel), we find that the change of sign takes place near the peak of the radiance.

A direct interpretation of such a velocity jump is that the spicule material is seen falling after rising. The velocity jump corresponds to locations where a "falling after rising" process is also indicated by the expansion front in the time-series radiance map. Note that the velocity often begins with a positive sign which means a red shift (i.e. away from the observer). This can be naturally interpreted as being due to the spicules tilting towards the back side of the sky plane; in that case the Doppler shift is red when the material rises and blue when it falls. The opposite situation will happen if the blue shift is first observed, i.e. the spicules tilt towards the observer.

In order to see in more detail the Doppler velocity behaviour in spicules, we have chosen another dataset in which the line is cooler ( $\mathrm{C}$ III $977 \AA$ at $\approx 60000 \mathrm{~K}$ ) and stronger, so that the exposure time is only $15 \mathrm{~s}$. Although this dataset lasts only for $\approx 15 \mathrm{~min}$, we are able to see a better time resolution "close-up" of the Doppler velocity in spicules. In Fig. 6, we plot the radiance map and Dopplergram of the $\mathrm{C}$ III line obtained in PCH $2 b$, where we find a clear periodic variation of the Doppler shift. The period is around $5 \mathrm{~min}$, present at all heights between the limb and 15" above it.

Regarding the spicule periodicity, a recent observational and modeling study exhibited evidence for a relationship between the $\mathrm{H} \alpha$ spicule occurrence in an active region and persistent photospheric oscillation (De Pontieu et al. 2003, 2004).

\section{Long macro-spicules}

In the presently studied datasets, there are spicules that reach lengths higher than $30^{\prime \prime}$ off-limb (see Figs. 2 and 3). As discussed in the introduction, we refer to these with the term "long macro-spicule". We have looked at the evolution of some of these long macro-spicules. In Figs. 7 and 8 we plot the timeseries radiance and LOS velocity maps, as derived from the N IV line characteristics, for the four longest macrospicules from $\mathrm{PCH} 1$ and $\mathrm{PCH} 2 \mathrm{a}$.

During the observations, the SUMER slit was pointing to the center of the Sun's poles across the limb. Thus, it practically followed the same region on the Sun for the whole observing time, with the rotational compensation switched off. The instrument itself had no mechanical movement between any two exposures. In addition, the SoHO spacecraft has a good pointing stability better than $1^{\prime \prime}$ over a 15 min interval (Domingo et al. 1995). Therefore, we believe that the features displayed here were actually from a specific part of a spicule. When looking at such detailed plots of long macro-spicules one can see how the radiance of this specific location varies along their lifetime, as well as the behaviour of the Doppler velocity.

The time-series radiance maps shown in Figs. 7 and 8 reveal that long macro-spicules are highly structured. In the first frame of Fig. 7, two bright columns with bright feet at the same location near the limb can be identified between $0^{\prime \prime}$ and $-10^{\prime \prime}$, centered at $473 \mathrm{~min}$ and $478 \mathrm{~min}$, respectively. The time interval between them is about $5 \mathrm{~min}$. Another bright column between $-5^{\prime \prime}$ and $-15^{\prime \prime}$ centered at 481 min seems to disconnect 

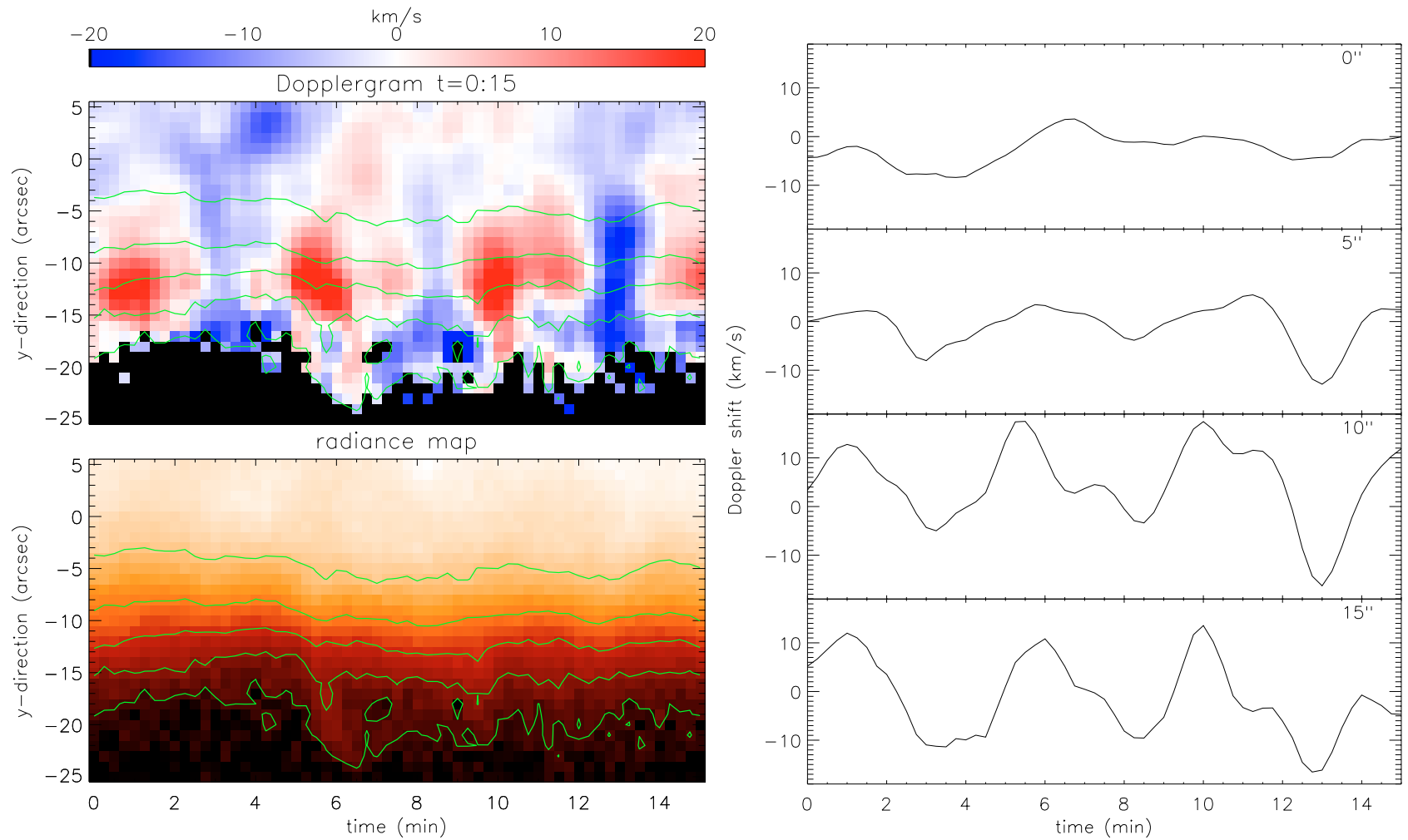

Fig. 6. Time-series Dopplergram and radiance map for the C III $977 \AA ̊$ line, in PCH 2b, with contours showing levels of radiance in logarithmic scale. Right panels show the relative Doppler shift at four locations above the limb: at $0^{\prime \prime}, 5^{\prime \prime}, 10^{\prime \prime}$ and $15^{\prime \prime}$ off-limb.
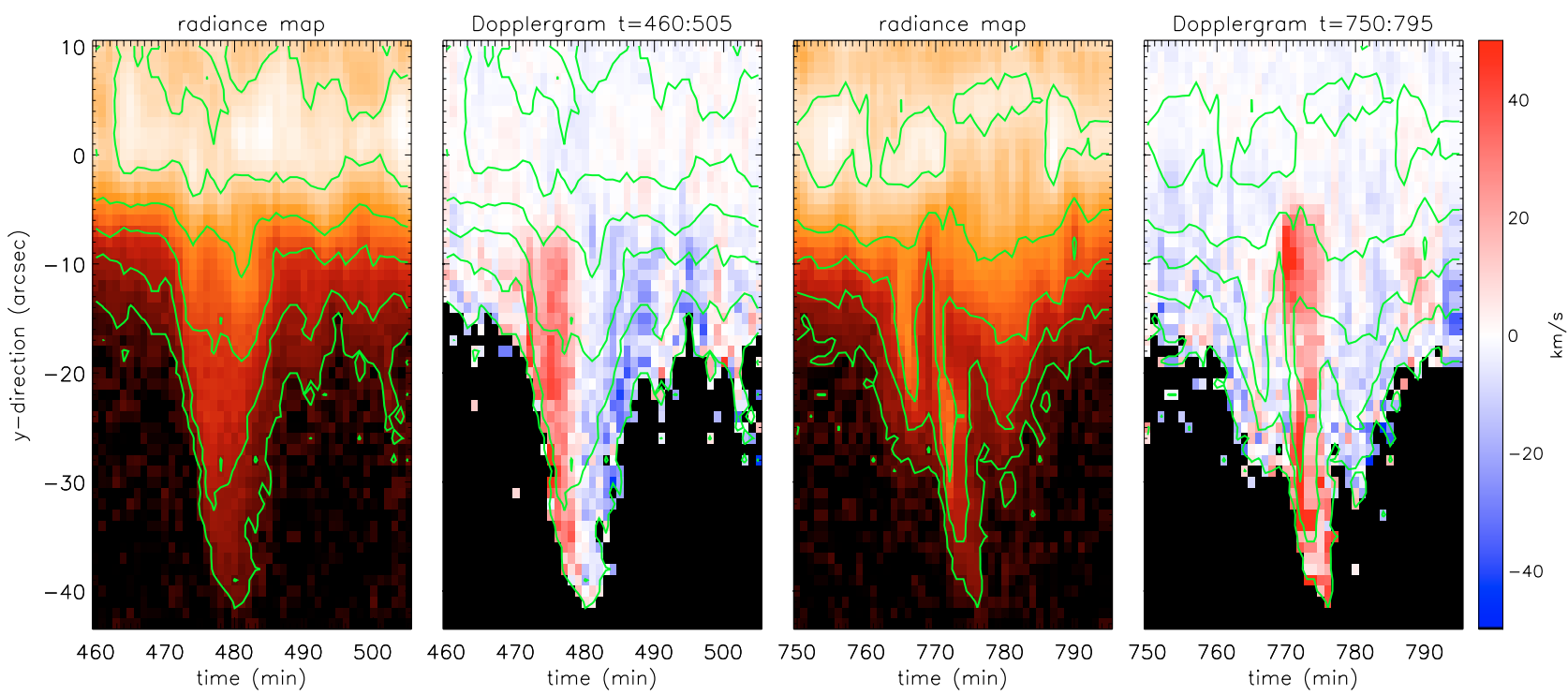

Fig. 7. Time-series radiance map and Dopplergram in the N IV $765 \AA$ line of two long macrospicules taken from PCH $2 \mathrm{a}$. The plotted time intervals are between 460 and $505 \mathrm{~min}$ (left two panels) and 750 and $795 \mathrm{~min}$ (right two panels). The overlaid contours show radiance in logarithmic scale.

with the low-lying bright features. We cannot know whether it is another jet or just the returning plasma of the macrospicule. A similar situation can be found at the leading edges of the other long macro-spicule, but less obvious concerning the 5-min interval for the one centered at $90 \mathrm{~min}$ plotted in Fig. 8. For the macro-spicule centered at $775 \mathrm{~min}$ in Fig. 7, individual components from its right side seem to have different footpoints (a few arcsec away from those on the left).
The fact that long macro-spicules are composed of several individual spikes seems to be a general characteristic for both datasets from PCH 1 and PCH 2a (look again at Fig. 2, upper panel, left and Fig. 4). Some of them have an obvious periodicity in the radiance of $\approx 5 \mathrm{~min}$, regardless of the exposure time of the dataset. This corresponds exactly to the average lifetime of a "normal" spicule, as seen in the same data. This therefore suggests that a long macro-spicule is made from a group of 


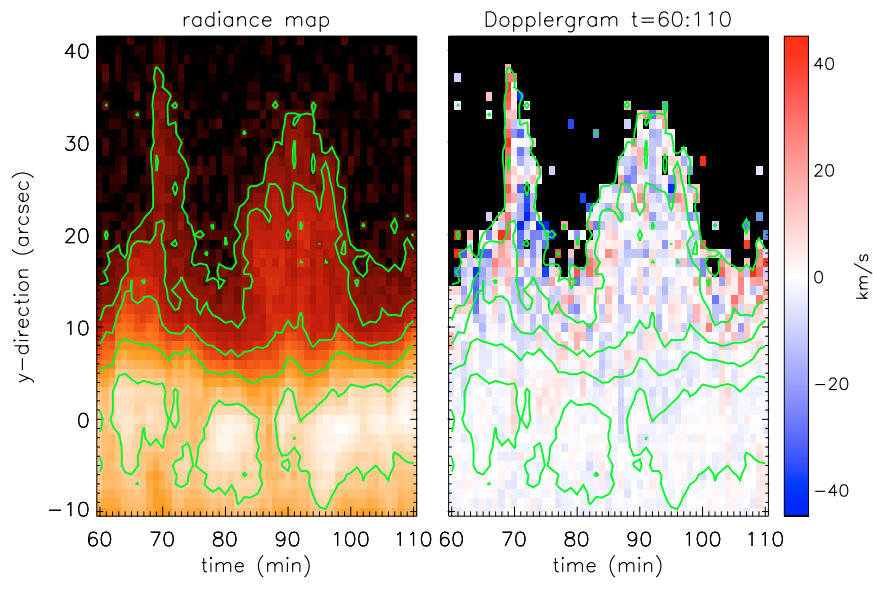

Fig. 8. The same as Fig. 7, but taken from PCH 1 (the time interval between 60 and $110 \mathrm{~min}$ ).

spicules that reach larger heights. It is also possible that two adjacent components could actually represent the same plasma loop, tilting backward and then towards us (or vice-versa), but this requires a special configuration: the loop to be oriented along the line of sight, with both feet inside the slit.

One finds, in the time series Dopplergrams shown in Figs. 7 and 8, that the amplitude of the Doppler shift variation for the macro-spicules is the smallest (mostly ranging from $\approx-5$ to $+5 \mathrm{~km} \mathrm{~s}^{-1}$ ) just above the limb. The Doppler shift values quickly increase with height above $10^{\prime \prime}$, reaching a maximum between $20^{\prime \prime}$ and $30^{\prime \prime}$. The maximum red-shift ranges from $\approx 20$ to $60 \mathrm{~km} \mathrm{~s}^{-1}$, while the blue-shift from $\approx-10$ to $-40 \mathrm{~km} \mathrm{~s}^{-1}$. As pointed out before when we discussed Fig. 5, the precision of the Doppler shift drops together with the plasma density, therefore we can only trust its values inside spicules. At large offlimb positions outside spicules, the derived velocity values are not reliable, and thus they have not been plotted.

The two long macro-spicules from Fig. 7 and the left one in Fig. 8 are found to be composed of several increases in radiance (individual spicules) with alternating velocity signs. On the contrary, the one centered at $92 \mathrm{~min}$ in Fig. 8 exhibits a different Doppler pattern, without a similar systematical change of the velocity signs. A possible reason may be that this macro-spicule was located in the sky plane, so no systematical LOS velocity was detected.

Long macrospicules are often observed having one side with a red-shift and another side with a blue-shift if seen in the Dopplergrams obtained by CDS/SoHO (see, e.g. Pike \& Mason 1998; Banerjee et al. 2000). This has been interpreted as evidence of rotating motion of the spicular plasma, which may be driven by torsional Alfvén waves propagating along an open magnetic flux tube (Kudoh \& Shibata 1999). Another possible explanation is that this would be an indication of a flow up and down along a loop with both feet rooted on the Sun's surface. In that case, its projection constructs a spike-like structure in the sky plane.

In our temporal series data, we see that the long macrospicule consists of several increases in radiance (individual spicules) with alternating Doppler velocity signs. This phenomenon could be caused by an up-going followed by a returning of the macro-spicule's plasma or just simply by individual spicules if they tilted away from the sky plane. An interpretation as a flow along a loop seems to require a special geometrical configuration, as discussed above. Furthermore, we emphasize here that the transition of Doppler shift actually took place at the same location, on top of the peak. Should a rotating motion have been considered, we would have had an interesting result that the transverse velocity changes its sign during the decaying phase of macro-spicules. It is also possible that, as suggested by Pike \& Mason (1998), a combination of both rotating and up-going motion was indeed observed. Therefore, it seems that at the time being we are unable to get an exclusive interpretation, and a fast-repeated raster-scanning observation should hopefully provide a clear view on the time evolution of an entire spicule-structure.

The low velocity in the early stage of spicule evolution deduced from the line shift has also been found with CDS observations (Pike \& Harrison 1997; Pike \& Mason 1998). The reason why spicules have a low Doppler velocity just above the limb is unclear. One possible explanation is that the measured line profiles are contributed largely by the background emission there, which stems from surrounding structures being at rest. From a statistical point of view, velocities from unresolved spicules with random orientations may also cause a small total shift by cancellation with each other. Another possibility is that spicules are really undergoing acceleration at these heights, as suggested by Pike \& Harrison (1997). We believe that a further study is necessary in order to answer this question. In the radiance map from Fig. 3, many bright structures show a vertical appearance which might reveal that they are ejected quickly in this initial stage, an idea consistent with previous studies (Karovska \& Habbal 1994; Parenti et al. 2002).

\section{Summary and conclusions}

In this study we analysed the behaviour of solar EUV spicules in three observing sequences, one of them being as long as $\approx 14 \mathrm{~h}$. It is of great interest to see the spicules continuously appearing and then fading away at the same location, inside both the northern and the southern polar coronal holes.

Most spicules studied here have a height between $10^{\prime \prime}$ and $20^{\prime \prime}$ above the limb. Nevertheless, a few of them can reach a height up to 40 ", being defined then as "long macro-spicules". Their lifetime ranges from $15 \mathrm{~min}$ down to $\approx 3 \mathrm{~min}$. These results are consistent with previous studies carried out by various authors cited in the introduction.

We have concluded that EUV spicules occur repeatedly with a birth rate of around $0.16 / \mathrm{min}$ as estimated at $10^{\prime \prime}$ above the limb. But their periodicity is difficult to determine, as spicules have different heights and it is not always possible to distinguish individual features. Nevertheless, a careful examination of the long datasets in the N IV line, as well as a short but better resolution data in the $\mathrm{C}$ III line indicate that the periods would be around $5 \mathrm{~min}$.

Another conclusion derived is that the high long-lived macro-spicules are not a single entity, but a group of plasma jets reaching larger heights, occurring repeatedly at a time interval similar to the normal spicules, of about $5 \mathrm{~min}$. 
A fast-repeated raster-scanning observation is indeed necessary to provide a clearer view on the time evolution of a spicule, and especially to what produces the red-blue velocity structure clearly seen in long macro-spicules.

Acknowledgements. Research at Armagh Observatory is grant-aided by the N. Ireland Dept. of Culture, Arts and Leisure. This work was supported in part by PPARC grant PPA/G/S/2002/00020 and by the Programme for Research in Irish Third Level Institutions for Grid-enabled Computational Physics of Natural Phenomena (Cosmogrid). The work of LDX in China was supported by the Key Program of the Chinese Academy of Science (KZCX3-SW-144). The SUMER project is financially supported by DLR, CNES, NASA and the ESA PRODEX programme (Swiss contribution). We thank the EIT team for the full-Sun data. Finally, we thank the referee for the helpful comments and suggestions.

\section{References}

Banerjee, D., O’Shea, E., \& Doyle, J. G. 2000, A\&A, 355, 1152

Beckers, J. M. 1968, Sol. Phys., 3, 367

Beckers, J. M. 1972, ARA\&A, 10, 73

Bohlin, J. D., Vogel, S. N., Purcell, J. D., et al. 1975, ApJ, 197, L133

Budnik, F., Schröder, K.-P., Wilhelm, K., \& Glassmeier, K.-H. 1998, A\&A, 334, L77

Dammasch, I. E., Wilhelm, K., Curdt, W., \& Hassler, D. M. 1999, A\&A, 346, 285
De Pontieu, B., Erdélyi, R., \& de Wijn, A. G. 2003, ApJ, 595, L63

De Pontieu, B., Erdélyi, R., \& James, S. P. 2004, Nature, 430, 536

Dere, K. P., Bartoe, J.-D. F., \& Brueckner, G. E. 1983, ApJ, 267, L65

Dere, K. P., Bartoe, J.-D. F., Brueckner, G. E., \& Recely, F. 1989, ApJ, 345, L95

Domingo, V., Fleck, B., \& Poland, A. I. 1995, Sol. Phys., 162, 1

Karovska, M., \& Habbal, S. 1994, ApJ, 431, L59

Kudoh, T., \& Shibata, K. 1999, ApJ, 514, 493

Lemaire, P., Wilhelm, K., Curdt, W., et al. 1997, Sol. Phys., 170, 105

Mariska, J. T. 1992, Cambridge Astrophys. Ser., The Solar Transition Region (Cambridge Univ. Press), 23

Moore, R. L., Tang, F., Bohlin, J., \& Golub, L. 1977, ApJ, 218, 286

Parenti, S., Bromage, B., \& Bromage, G. E. 2002, A\&A, 384, 303

Pike, C., \& Harrison, R. 1997, Sol. Phys., 175, 457

Pike, C., \& Mason, H. 1998, Sol. Phys., 182, 333

Popescu, M. D., Doyle, J. G., \& Xia, L. D. 2004, A\&A, 421, 339

Roberts, W. O. 1945, ApJ, 101, 136

Secchi, A. 1877, Le Soleil (Paris: Gauthier-Villars), 2

Sterling, A. C. 2000, Sol. Phys., 196, 79

Wilhelm, K. 2000, A\&A, 360, 351

Wilhelm, K., Curdt, W., Marsch, E., et al. 1995, Sol. Phys., 162, 189

Wilhelm, K., Lemaire, P., Curdt, W., et al. 1997, Sol. Phys., 170, 75

Wilhelm, K., Dammasch, I. E., Marsch, E., \& Hassler, D. M. 2000, A\&A, 353, 749

Withbroe, G. L. 1983, ApJ, 267, 825

Xia, L. D. 2003, Ph.D. Thesis, Georg-August-Universität, Göttingen 